\title{
The Crystallization Transformations Sequence as Ice is Compressed at Low Temperature: Avoidance of Amorphization
}

\author{
C Tulk ${ }^{1}$, J Molaison ${ }^{1}$, A Makhluf ${ }^{2}$, C Manning ${ }^{2}$, D Klug ${ }^{3}$ \\ ${ }^{1}$ Oak Ridge National Laboratory, Oak Ridge, TN, ${ }^{2}$ Department of Earth, Planetary and Space \\ Sciences, ${ }^{3}$ National Research Council of Canada \\ tulkca@ornl.gov
}

The compression of ice I is, as expected, seen to form high density amorphous (HDA) ice when compressed continuously to $\sim 1 \mathrm{GPa}$. However, when ice I is compressed at a slower rate, with breaks between 1 Atm and 1 $\mathrm{GPa}$, the formation of HDA can be avoided. Therefore resulting in the formation of a series of crystalline states. This demonstrates the crystalline-to-crystalline transformation sequence of the lowest energy structures when the amorphization of ice Ih into the HDA form is avoided. An explanation of the structural complexities of the crystalline transformation sequence indicate that high density amorphous ice is a result of an interrupted crystal-tocrystal transition between ice I and ice VIII, and is likely not a 'melting' process to a super-cooled liquid. Additionally, new data confirming the accurate calibration of our temperature measurement is provided, in addition to new thermal pathway analysis of the transformation sequence.

Acta Cryst. (2020). A76, a150 